\title{
PREVALENCE AND ANTIBIOTIC SUSCEPTIBILITY PATTERN OF PSEUDOMONAS SPECIES ISOLATED FROM CLINICAL SAMPLES IN A TERTIARY CARE HOSPITAL
}

\author{
M. ABDUR RAHMAN ${ }^{1}$, POOJA NAIR ${ }^{1 *}$ \\ ${ }^{1}$ Department of Microbiology, Saveetha Medical College and Hospital, Thandalam, Chennai \\ "Email: poojarnair2007@gmail.com
}

Received: 12 Sep 2020, Revised and Accepted: 15 Nov 2020

\begin{abstract}
Objective: Pseudomonas aeruginosa is the commonest agent causing opportunistic nosocomial infections, contributing to mortality and morbidity worldwide. Along with its intrinsic and acquired resistance mechanisms, infections caused by Pseudomonas species further lead to treatment failure. This study was done to determine the prevalence and antimicrobial susceptibility pattern of different species of Pseudomonas isolated from various clinical samples by phenotypic methods.
\end{abstract}

Methods: This study involved the examination of clinical samples for various species of Pseudomonas by using different standard biochemical tests. Their antimicrobial susceptibility profile was performed by the Kirby Bauer disc diffusion method. Analysis of the antibiogram pattern was done to study the multi-drug resistance among Pseudomonas species.

Results: Out of the 1249 bacterial isolates, Pseudomonas species accounted for $12.9 \%$. Pseudomonas species showed maximum resistance to gentamicin (31.4\%), followed by ciprofloxacin (30.2\%. and showed the least resistance tocolistin and polymyxin B. 46 isolates out of the 162 (28\%) were found to be Multi-Drug Resistant (MDR) Pseudomonas aeruginosa.

Conclusion: The present study highlights that Pseudomonas species remains a major cause of hospital-acquired infections. Multidrug resistance was observed in most of the strains, which makes the therapeutic options more difficult. Surveillance of antimicrobial resistance and strict infection control measures are essentially to be practiced in managing and control of infections caused by Pseudomonas.

Keywords: Pseudomonas aeruginosa, Antimicrobial susceptibility, Multidrug resistance

(C) 2021 The Authors. Published by Innovare Academic Sciences Pvt Ltd. This is an open access article under the CC BY license (https://creativecommons.org/licenses/by/4.0/) DOI: https://dx.doi.org/10.22159/ijcpr.2021v13i1.40814. Journal homepage: https://innovareacademics.in/journals/index.php/ijcpr

\section{INTRODUCTION}

The genus Pseudomonas, being one of the most complex bacterial genera, contains more than 140 species, among which most of them are saphrophytic. Around 25 species are associated with humans to cause opportunistic infections. Some of the important clinically relevant species are P. aeruginosa, P. fluorescens, P. putida, P. stutzeri, P. mallei, P. pseudomallei, P. maltophilaand P. putrefaciens $[1,2]$. Pseudomonas aeruginosa is the most commonly identified one from the clinical specimens of hospital admitted patients [3]. Even though it is a commensal of microflora in healthy humans, it is a commonly encountered causative agent of infections seen in hospitalized patients, particularly in burns, respiratory diseases, catheterized and immunocompromised patients. It is one of the commonest gram-negative bacteria that takes advantage of an individual's weakened immune status to cause infection by its tissue-damaging toxins. Infections caused by Pseudomonas aeruginosa can infect any anatomical site; hence it can be isolated from various body fluids such as wounds, urine, blood, wounds, eye or ear swabs, and sputum. It is also a grave concern to cancer and burn patients. It can cause infections such as Urinary Tract Infections (UTI), respiratory infections, particularly Ventillator-Associated Pneumonia (VAP) in debilitated patients, bone and joint infections, dermatitis, otitis media, bacteremia and other numerous variety of systemic infections $[4,5]$.

It is ubiquitous in nature. It has the ability to thrive and colonize anywhere, being widely distributed in the environment-soil, vegetation, water bodies, sewage, hospitals and even on the moist sites of the skin of healthy individuals. And its ability to resist the antibacterial and antiseptic agents makes it more complex. This organism is hard to treat because of its acquired and intrinsic resistance $[1,6]$. The defensemechanism of Pseudomonas aeruginosa makes it immune to many antibiotics by different means such as chromosomally encoded genes, restricted outer membrane permeability, production of antibiotic inactivating enzymes or by the efflux system that pumps antibiotics out of the cell $[7,8]$.

Keeping in view the knowledge about the ability of Pseudomonas spp. to thrive in myriad of habitat, its etiology as well as the pathology and its wide range of mechanisms of resistance to antibiotics, this study was aimed to isolate and characterize various Pseudomonas species from diverse clinical samples of a tertiary care hospital and to analyze its antibiotic susceptibility profile.

\section{MATERIALS AND METHODS}

This perspective cross-sectional study was conducted for a period of 3 mo from December 2019 to February 2020 in the clinical microbiology laboratory at Saveetha Medical College. Clinical samples received in the laboratory were subjected to gram staining and were inoculated on to blood agar and mac conkey agar. In addition to these, chocolate agar was used for culturing respiratory samples and blood. All the culture Medias were incubated aerobically at $37{ }^{\circ} \mathrm{C}$ overnight. Routine biochemical tests were used to identify Pseudomonas. These included colony morphology, pigment production, motility test, oxidase test, catalase test, growth at $41{ }^{\circ} \mathrm{C}$, nitrate reduction, gelatin hydrolysis, arginine dihydrolase and sugar fermentation test.

The isolated species were subjected to an Antibiotic susceptibility test by the Kirby Bauer disc diffusion method recommended by the Clinical Laboratory Standard Institute (CLSI) guidelines. Individual isolates were inoculated on Mueller Hinton Agar plates at a concentration of $10^{6}$ Colony Forming Units (CFU) by lawn culture method. Discs impregnated with antibiotics Amikacin (30 mcg), Gentamicin (10 mcg), Ceftazidime (30 mcg), Ciprofloxacin (5mcg), Imipenem $(10 \mathrm{mcg})$, Meropenem $(10 \mathrm{mcg})$, Ertapenem $(10 \mathrm{mcg})$, Ofloxacin (5mcg), Cefaperazonesulbactam $(75 / 30 \mathrm{mcg})$, Cefepime $(30 \mathrm{mcg})$, Piperacillintazobactam $(100 / 10 \mathrm{mcg})$, Tigecycline $(15 \mathrm{mcg})$, 
Colistin (10mcg) and Polymyxin B (300mcg) were placed. Plates were examined after $24 \mathrm{~h}$ of incubation at $37^{\circ} \mathrm{C}$.

\section{RESULTS}

During the study period, a total of 1249 bacterial isolates were obtained, in which 162 isolates were found to be Pseudomonas species.
Prevalence of Pseudomonas species was 12.9\%. Pseudomonas species were predominantly recovered from exudates (pus samples, wound swabs, tissues and body fluids) followed by respiratory samples (sputum, bronchoalveolar lavage, endotracheal aspirates), blood samples and urine. The distribution of clinical samples from which Pseudomonas species were isolated is given in fig. 1.

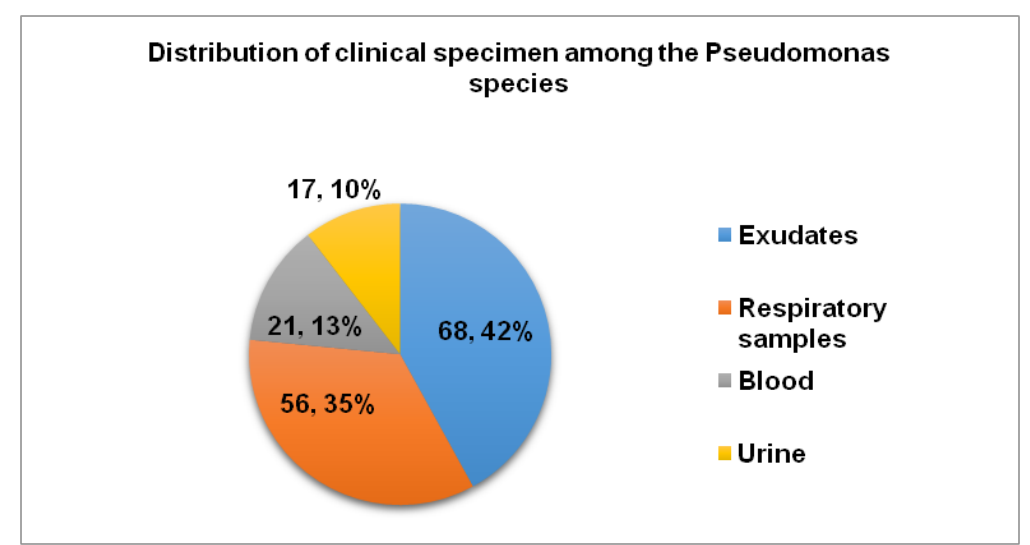

Fig. 1: Distribution of clinical specimen types among the Pseudomonas species isolated, pseudomonas isolates were more from male patients $(109 ; 67 \%)$ than from female patients $(53 ; 33 \%)$ [table 1$]$

Table 1: Distribution of male and female patients among IP-OP departments

\begin{tabular}{llll}
\hline Patients & IP & OP & Total \\
\hline Male & 98 & 11 & $109(67.3 \%)$ \\
Female & 47 & 6 & $53(32.7 \%)$ \\
Total & $145(90 \%)$ & $17(10 \%)$ & \\
\hline
\end{tabular}

Pseudomonal infections were observed highest in the age group 46-60 y (36\%) followed by 61-75 y (28\%) and 31-45 y (20\%). Age-wise distribution is given in table 2.

Table 2: Age-wise distribution of Pseudomonas isolates

\begin{tabular}{ll}
\hline Age group & No. of Pseudomonas spp isolated \\
\hline $0-15$ y & 1 \\
$16-30$ y & 18 \\
$31-45$ y & 33 \\
$46-60$ y & 58 \\
$61-75$ y & 45 \\
$76-90$ y & 7 \\
\hline
\end{tabular}

Almost $90 \%$ of the Pseudomonas species were found out from inpatients in which it was highest from surgical wards (19\%), ICU (17\%) followed by medicine wards (14\%) and Chest and TB medicine (10\%). Detailed distribution of Pseudomonas species from various other departments are given in the fig. 2.

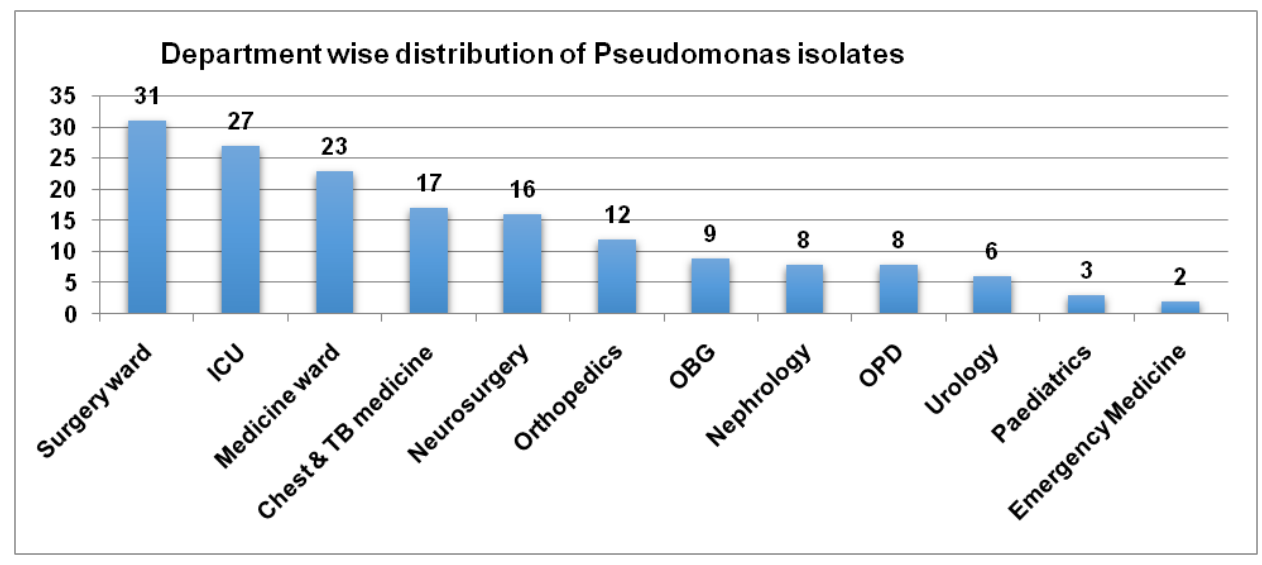

Fig. 2: Department wise distribution of Pseudomonas isolates 
Out of the total isolates, Pseudomonas aeruginosa was the most prevalent species, which accounted for $87 \%$ followed by Pseudomonas stutzeri (6\%) and Pseudomonas putida (4\%).
Pseudomonas fluorescens (2\%) and Pseudomonas maltophila (1\%) were the least isolated species (fig. 3: Distribution of various species of Pseudomonas isolated from clinical specimens).

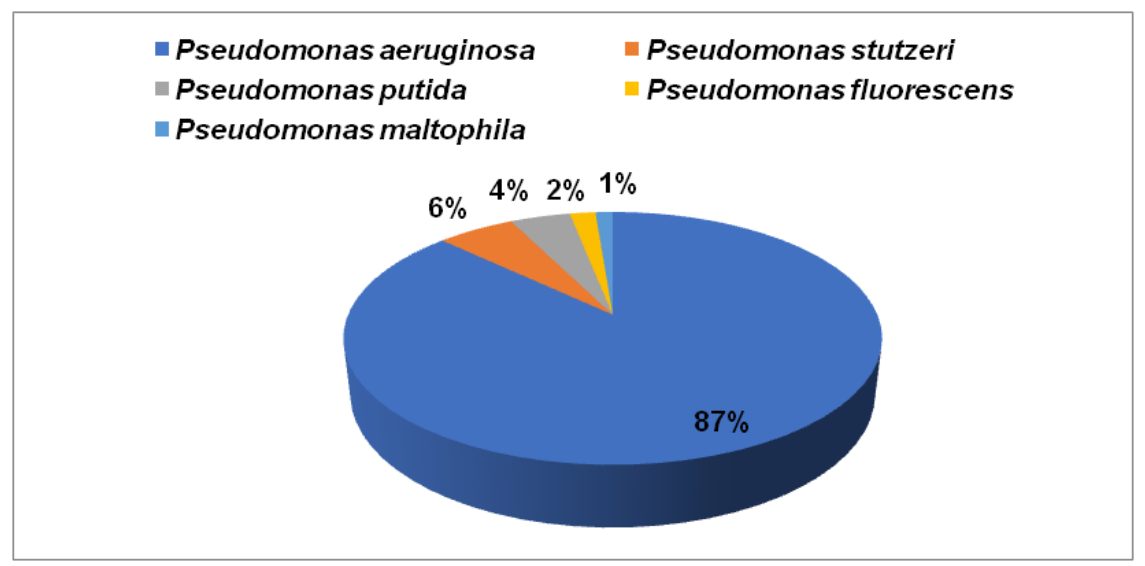

Fig. 3: Distribution of various species of Pseudomonas isolated from clinical specimens

Most of the strains were resistant to fluoroquinolones, aminoglycosides, cephalosporins and carbapenem group of drugs. The highest level of resistance was observed against Gentamicin, which is an aminoglycoside with 51 strains to be resistant, followed by ciprofloxacin, a fluoroquinolone group of drug (49, $30.2 \%)$, ceftazidime $(45 ; 27.7 \%)$ cefoperazone-sulbactam (41;25.3\%), amikacin (40;24.6\%), meropenem (31; 19.1\%), cefepime $(31 ; 19.1 \%)$, imipenem $(30 ; 18.5 \%)$, piperacillintazobactam $(29 ; 17.9 \%)$ and ertapenem $(27 ; 16.6 \%)$. Most of the strains showed sensitivity towards Colistin and Polymyxin B.

Forty-six strains (28\%) of Pseudomonas spp were multidrugresistant (MDR) i. e, strains resistant to three or more than three different group of drugs. And interestingly, all the 46 strains were Pseudomonas aeruginosa.

Table 3: Antimicrobial resistance pattern of Pseudomonas spp

\begin{tabular}{lll}
\hline Antibacterial drug & \% of isolates showing resistance & \% of isolates showing sensitivity \\
\hline Amikacin & $24.6 \%$ & $75.4 \%$ \\
Gentamicin & $31.4 \%$ & $68.6 \%$ \\
Ceftazidime & $27.7 \%$ & $72.3 \%$ \\
Ciprofloxacin & $30.2 \%$ & $69.8 \%$ \\
Imipenem & $18.5 \%$ & $81.5 \%$ \\
Meropenem & $19.1 \%$ & $80.9 \%$ \\
Ertapenem & $16.6 \%$ & $83.4 \%$ \\
Ofloxacin & $14.1 \%$ & $85.9 \%$ \\
Cefaperazone-sulbactam & $25.3 \%$ & $74.7 \%$ \\
Cefepime & $19.1 \%$ & $80.9 \%$ \\
Piperacillin-tazobactam & $17.9 \%$ & $82.1 \%$ \\
Tigecycline & $13.5 \%$ & $86.5 \%$ \\
Colistin & $3.1 \%$ & $96.9 \%$ \\
Polymyxin B & $3.7 \%$ & $96.3 \%$ \\
\hline
\end{tabular}

\section{DISCUSSION}

Pseudomonas aeruginosa is the most common gram-negative, nonfermenting bacteria isolated from clinical specimens that pose serious challenge for the treatment of nosocomial infections. The main objective of our study was to investigate the epidemiological data of Pseudomonas spp. isolated from various clinical samples and to analyze the antimicrobial resistance patterns against routinely used antibiotics.

In our study, highest percent (19\%) of Pseudomonas spp was obtained from surgery units, which is in concordance with the result of Sharma et al. [3] with $33 \%$. Most of the patients infected by Pseudomonas spp are male $(109 ; 67.3 \%)$ when compared to the females, which is in close concordance with the study of Josef et al. [6], (113 males; 67.3\%). Polymyxin B and colistin shows the highest percentage of sensitivity and percentage of resistance being low $(3.7 \%, 3.1 \%)$, which also supports other studies that are made throughout India, making them the ideal antimicrobial agent. 46 Pseudomonas (28\%) were MDR strains. Several studies with similar MDR rates were reported-24.4\% by Josef et al. [6], $25.35 \%$ by
Qayoom et al. [9] and 24\% by Sharma et al. [3]. High percentage of MDR isolated from clinical samples is worrisome. To contain the spread of drug resistance, appropriate laboratory detection, control of patient-to-patient transmission and judicious use of antibiotics should be done.

\section{CONCLUSION}

It is inevitable that the antibiotic susceptibility pattern of bacterial pathogens like $P$. aeruginosa in specialized clinical units should be continuously monitored so as to minimize the resistance to in use routine antibiotics. Judicious usage of antibiotics and creating a standard antibiotic policy that supports the clinician to choose the appropriate drug of choice helps in preventing drug resistance, whereas proper infection control measures and timely identification of resistant pathogen can help in reducing the spread of multidrugresistant strains.

\section{FUNDING}




\section{AUTHORS CONTRIBUTIONS}

All the authors have contributed equally.

\section{CONFLICT OF INTERESTS}

Declared none

\section{REFERENCES}

1. Palleroni NJ. Introduction to the family pseudomonadaceae. In Starr MP, Stolp H, Trüper HG, Balows A, Schlegel HG. (eds) The Prokaryotes. Springer, Berlin, Heidelberg; 1981.

2. Swaraj Mohanty, Bighneswar Baliyarsingh, Suraja Kumar Nayak. Antimicrobial resistance in Pseudomonas aeruginosa: a concise review; 2019. DOI:10.5772/intechopen.88706

3. Jaswinder Sharma, Surindersingh, Amariitkaur Gill, Amandeepkaur. Prevalence and antimicrobial susceptibility pattern of Pseudomonas aeruginosa isolated from pus samples in a tertiary care Hospital, Bathinda. Int J Contemporary Med Res 2016;3:3481-3.

4. Temesgen Bekele, Amene Tesfaye, Hebtewold Deti Waktola. Pseudomonas aeruginosa isolates and their antimicrobial susceptibility pattern among catheterized patients at Jimma
University Teaching Hospital, Jimma, Ethiopia. BMC Res Notes 2015;8:488.

5. Kiran A Deshmukh, Deepak Manthale. Prevalence and antibiotic susceptibility of Pseudomonas aeruginosa isolated from chronic suppurative otitis media. Int J Otorhinolaryngol Head Neck Surg 2017;3:56-60.

6. Josef Yayan, Beniam Ghebremedhin, Kurt Rasche. Antibiotic resistance of Pseudomonas aeruginosa in pneumonia at a single university hospital center in germany over a 10 Y Period. PLoS ONE 2015;10:e0139836.

7. Tarig MS Alnour, Eltayib Hassan Ahmed Abakur. Multidrugresistant Pseudomonas (P) aeruginosa: medical impact, pathogenicity, resistance mechanisms and epidemiology. JSM Microbiol 2017;5:1046.

8. Alain C Juayang. Five-year antimicrobial susceptibility of Pseudomonas aeruginosa from a local tertiary hospital in Bacolod City, Philippines. Trop Med Infect Dis 2017;2:28.

9. Sumaira Qayoom, Afreen Rashid, Amrish Kohli, Talat Masoodi, Muzafar Amin. Prevalence and antibiotic sensitivity pattern of Pseudomonas aeruginosa isolate from respiratory samples, pus samples and body fluids in a tertiary care hospital, Kashmir. Indian J Microbiol Res 2019;6:345-9. 